\title{
Think outside the sandbox
}

\author{
One grain of sand is a solid. But a lot of grains together can behave \\ like a solid or a liquid. By probing this dual personality, physicists hope \\ to understand a host of real-world systems, says Mark Buchanan.
}

$\mathrm{H}$ einrich Jaeger knows what it's like, literally and metaphorically, to watch the sand slipping through the hourglass. "We thought it would be a four-week experiment," the physicist recalls of one of his studies. "It ended up taking several years."

A decade ago, Jaeger and his colleagues at the University of Chicago were busy studying what happened when they poured sand into a container and tapped the box repeatedly. They expected the grains to bed down fairly quickly — but, as the team eventually reported in 1995, the sand kept shifting into ever more compact configurations. " "Granular systems look deceptively simple," says Jaeger. "But the closer we look, we find so many complexities."

Such puzzles show why granular materials hold a special fascination for physicists interested in the fundamental properties of matter. A pile of sand, flour or mustard seed can stand tall and fixed like a solid, defying the tug of gravity that would bring water sloshing to the floor. Yet sand pours through an hourglass as though it were a liquid.

People spend more time and effort in handling granular matter than any type of material aside from water. In industry, powders clog chutes and otherwise misbehave, at enormous economic expense. So understanding how simple forces - mostly gravity and friction - help to shape the materials' multiple personalities is of immense practical significance.

\section{Out of equilibrium}

What's more, some physicists believe that progress towards a theory of granular matter could provide clues to the wider world of 'non-equilibrium' physics. This is a large missing piece of the theoretical framework that physicists use to describe matter, and applies to the vast majority of real-world systems, which are not held in fixed and unchanging conditions but instead are shaken, stirred, heated, or driven by energy in countless other ways.

Theorists have long approached granular matter by considering it to be analogous to ordinary matter, with the grains playing the roles of individual molecules ${ }^{2}$. At rest, a pile of grains is indeed rather like a solid, able to resist a certain amount of stress. But if you shake it vigorously, letting the grains tumble past one another and rearrange themselves, it does appear to shift into a

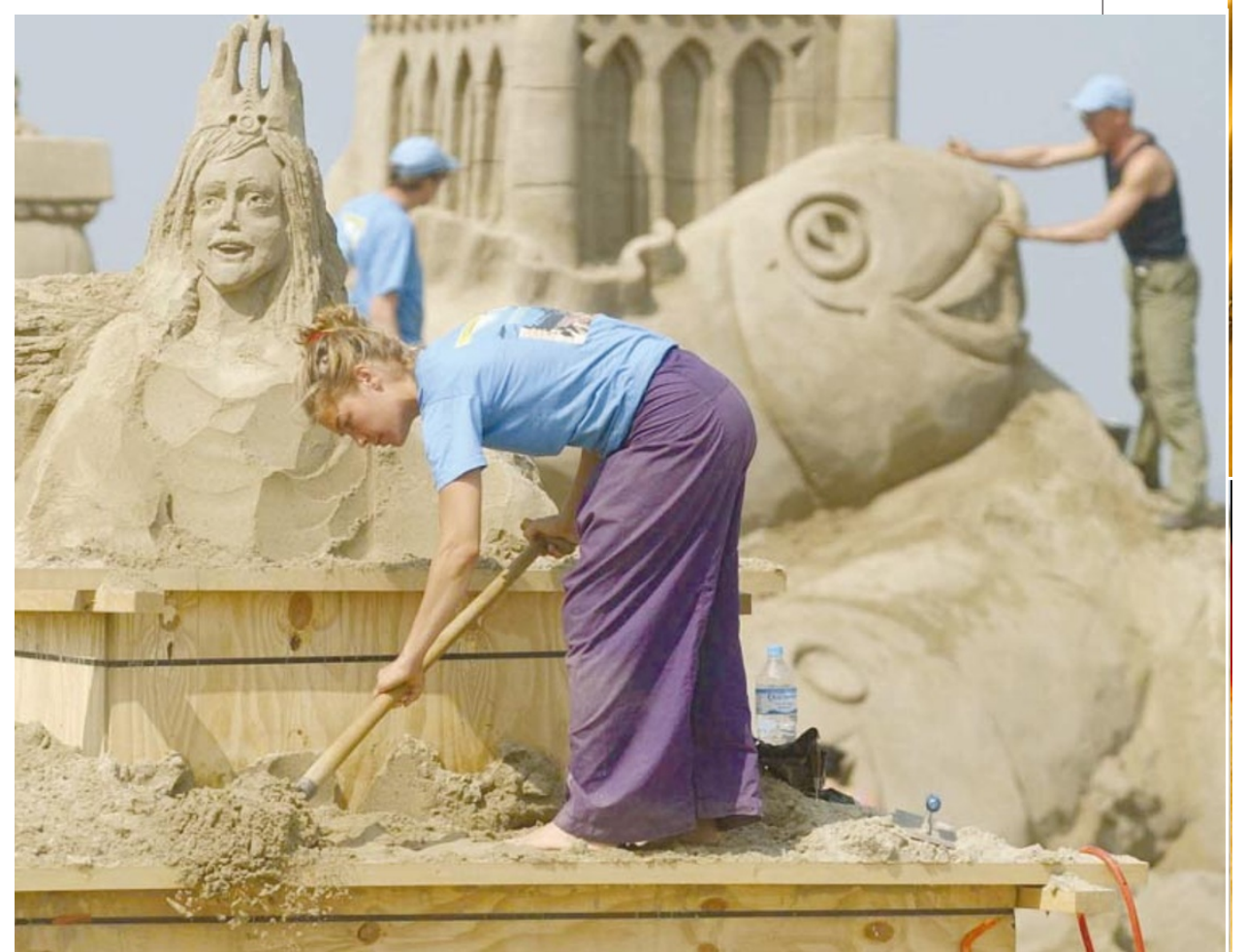

Stick or slick? Sand can be sculpted into solid forms, both by humans (above) and by the elements (above right). But put it in an hourglass and it flows like a liquid — and theorists want to know why.

'liquid' form. Much theorizing about the behaviour of granular matter therefore centres on the extent to which it is meaningful to talk about 'melting' piles of sand, and whether the degree of shaking might be related to an 'effective temperature' of the granular liquid.

Recent experimental results have revealed some fundamental similarities between granular liquids and their conventional counterparts. High-school physics students are familiar with the concept of brownian motion, in which a small particle floating in a pool of liquid dances around, driven by the thermal motions of the surrounding molecules. And Albert Einstein showed that the speed with which the particle wanders is linked to how easily the same particle can be dragged through the liquid by an external force ${ }^{3}$. Two numbers - a 'diffusion coefficient', $D$, and a 'mobility', $\mu-$ describe these two behaviours, and their ratio is always equal to the temperature of the liquid, $T$.

In August this year, Gianfranco D'Anna and his colleagues at the Swiss Federal Poly- technic in Lausanne showed that this relationship also holds true for granular liquids ${ }^{4}$. They put 50,000 glass beads in a small container, which they vibrated at high frequency to generate 'liquid' behaviour. The team used this set-up to run a simplified version of Einstein's experiment: whereas he considered a particle that could wander freely, the cone-shaped 'particle' used by D'Anna's team was able only to rotate.

\section{Go with the flow}

Whatever the size of the cone, the ratio between $D$ and $\mu$ was constant. And this effective temperature seemed to reflect accurately the vigour of the disordered motion of the constituent particles — just as a thermal temperature does for an ordinary liquid. "A granular liquid is indeed a fairly good liquid," says D’Anna.

The team's results lend support to the idea that granular matter is not such a misfit after all. They also raise an obvious question: if adequate vibration generates a liquid, what happens if the vibration is less strong? Weaker vibration corresponds to a lower 

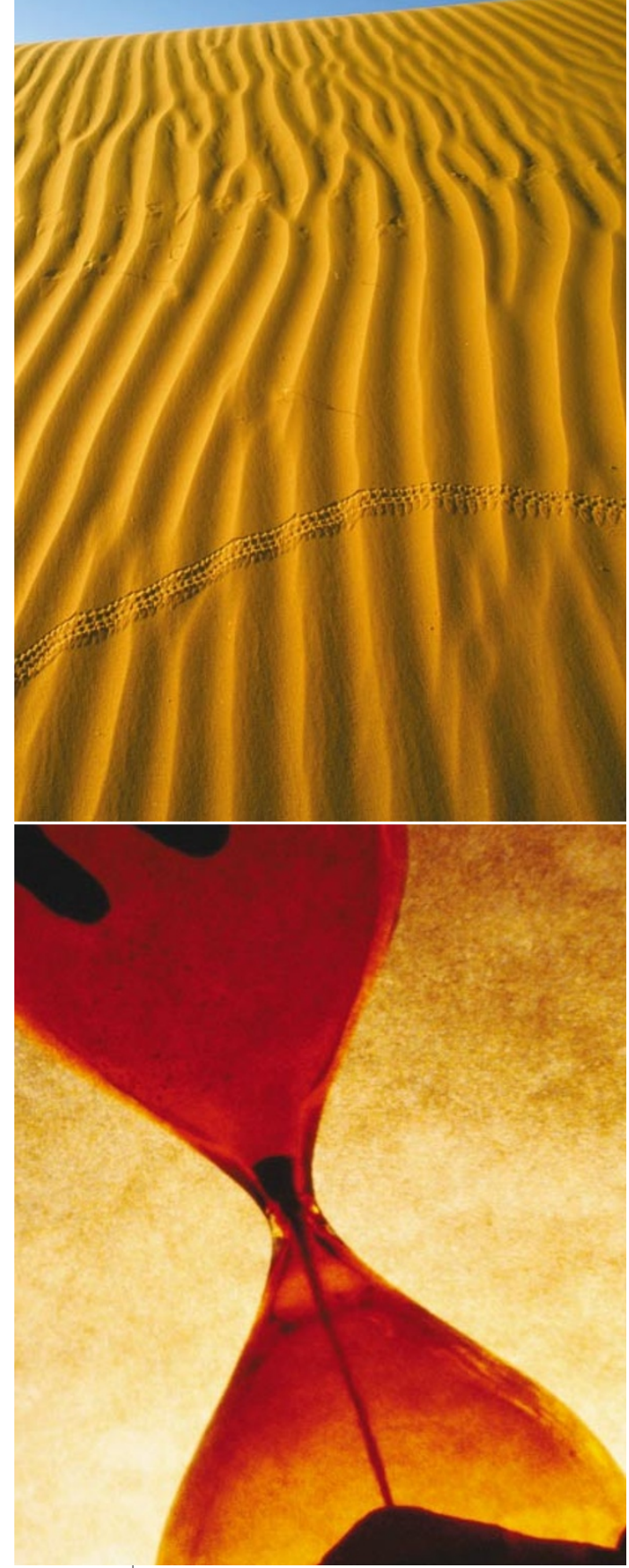

effective temperature. So, by analogy, a progressively less vigorously shaken granular liquid should reach a point at which it 'freezes' into a solid.

These are the conditions explored by Jaeger and his colleagues in their studies during the early 1990s. But their grains did not freeze into anything like an ordinary solid. Contrary to expectations, a few days of tapping did not bring the container of sand into a fully compact state. In some cases, the grains remained unsettled even after several hundred thousand taps ${ }^{1}$. As Jaeger's group suggested, this is similar to the way in which glassy materials solidify.

Upon cooling, the molecules of a glassy material do not fall into a regular, crystalline arrangement, but instead become jammed in a disordered structure. The molecules still

move, but with great difficulty. Because these structural rearrangements occur so slowly, the material is stable for most practical purposes. However, the physical properties of the glass - such as its electrical conductivity - gradually change as its structure evolves. Ordinary matter in equilibrium does not experience this sort of 'ageing'.

Since Jaeger's team reported its first results, theories developed to describe both granular solids and glassy materials have begun to converge - suggesting that the two types of matter are fundamentally similar.

\section{Grains of truth}

In the late 1980s, Sam Edwards of the University of Cambridge, UK, tried to formulate a theory of granular matter for one special case - a dense collection of grains flowing just fast enough to avoid sticking. For this regime, Edwards proposed that an accurate theory could be devised by supposing that the grains are equally likely to fall into any of the possible 'jammed' configurations ${ }^{5}$.

At the time, most physicists were unconvinced by the theory - it seemed possible, if not likely, that some of the jammed configurations would be more probable than others. "It was a very bold proposal," says Jorge Kurchan of the Ecole Supérieure de Physique et de Chimie Industrielles in Paris. "Few took it very seriously." A theory as simple as Edwards', physicists reasoned, was unlikely to explain the complexities of granular behaviour.

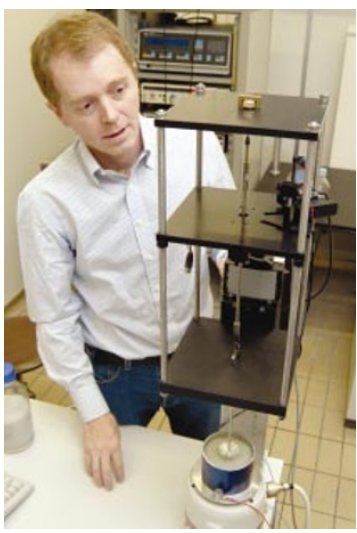

Gianfranco D'Anna has studied the physics of 'granular liquids'.

granular liquids, in which the grains leave their jammed configurations behind. And some physicists wonder about the utility of the 'effective temperature' concept. "It is not clear," says Douglas Durian of the University of California, Los Angeles, "if these temperatures have a predictive significance." In earlier experiments, for example, effective temperatures measured for different types of flow tended to have different values, whereas a truly fundamental temperature should give the same value no matter how it is measured.

But the fact that some of the same fundamental ideas seem to apply to both granular matter and glassy materials is encouraging, as both are examples of non-equilibrium systems - and gaining a general understanding of these systems is near the top of the 'to do' list for theoretical physicists.

Current theories of solids, liquids and gases are based on equilibrium statistical mechanics, which apply only to systems that are in thermal equilibrium. A bowl of water at room temperature is one such system, as it has the same temperature as its surroundings. But a granular liquid is not in equilibrium — it needs a constant input of vibrational energy to retain its 'liquid' state. A 'jammed' granular system is also out of equilibrium, as the grains cannot easily explore all configurations. Similarly, the way in which the character of glass evolves with time is a direct consequence of the system having excess energy and taking an

By the late 1990s, however, Kurchan and other theorists were developing ways to understand ageing in glasses in fundamental terms ${ }^{6}$ - and their theories bore a striking resemblance to Edwards' theory of granular matter. "In retrospect, his ideas were not so crazy after all," says Kurchan.

Last year, Kurchan teamed up with Hernán Makse of the City College of New York to test Edwards' ideas directly. Using a computer, they simulated the flow of grains trapped between a fixed lower surface and an upper surface moving just fast enough to keep the grains flowing. Like D'Anna and his colleagues, Kurchan and Makse measured the effective temperature of the grains by examining the relationship between $D$ and $\mu$ for a simulated probe particle. Kurchan and Makse also calculated the effective temperature using Edwards' theory, and found that the two results matched ${ }^{7}$.

Nonetheless, a full theory of granular matter remains some way off. As Kurchan points out, Edwards' ideas do not apply to incredibly long time to reach equilibrium.

The problem, for physicists interested in the fundamental behaviour of matter, is that most real-world systems are not in equilibrium. From the chemistry of living cells to the river of cars on a crowded highway, the real world is out of balance, perpetually flowing and evolving. If the recent surge of interest in granular matter leads to a deeper understanding of non-equilibrium systems in general, the theoretical pickings could be rich indeed. "Intellectually," says Jaeger, "things are beginning to gel."

\section{Mark Buchanan is a freelance writer based in}

Cambridge, UK.

1. Knight, J. B., Fandrich, C. G., Lau, C. N., Jaeger, H. M. \&

Nagel, S. R. Phys. Rev. E 51, 3957-3963 (1995).

2. Jaeger, H. M., Nagel, S. R. \& Behringer, R. P. Rev. Mod. Phys. 68, 1259-1273 (1996).

. Einstein, A. Ann. Physik 17, 549-560 (1905)

4. D'Anna, G., Mayor, P., Barrat, A., Loreto, V. \& Nori, F. Nature 424, 909-912 (2003).

5. Mehta, A. \& Edwards, S. F. Physica A 157 1091-1097 (1989)

6. Cugliandolo, L. F., Kuchan, J. \& Peliti, L. Phys. Rev. E 55, 3898-3914 (1997)

Makse, H. A. \& Kurchan, J. Nature 415, 614-616 (2002). 